\title{
Biomedical and Pharmacology Journal
}

\author{
Article Withdrawal Form
}

\section{Manuscript Title:}

\section{Reasons for withdrawal}

(All authors must sign this form in English)

Printed Name Signature

Email Address

Date

*

*(Correspondence Author)

Biomedical and Pharmacology Journal

10, Gulshan-e-Iqbal, New Maulana Azad Colony, Idgah Hills, Bhopal - 462001 Madhya Pradesh, India Website: biomedpharmajournal.org/ 